\title{
Electron-Impact Excitation Cross Sections for Modeling Non-Equilibrium Gas
}

\author{
Winifred M. Huo ${ }^{\mathrm{a}}$ \\ NASA Ames Research Center, Moffett Field, CA 94035, USA \\ Yen Liu $^{\mathrm{b}}$ \\ NASA Ames Research Center, Moffett Field, CA 94035, USA \\ Marco Panesi ${ }^{\mathrm{c}}$ \\ University of Illinois, Urbana-Champaign, IL 61801, USA \\ Alessandro Munafo ${ }^{\mathrm{d}}$ \\ University of Illinois, Urbana-Champaign, IL 61801, USA \\ Alan Wray ${ }^{\mathrm{e}}$ \\ NASA Ames Research Center, Moffett Field, CA 94035, USA \\ Duane F. Carbon ${ }^{\mathrm{f}}$ \\ NASA Ames Research Center, Moffett Field, CA 94035, USA
}

\begin{abstract}
In order to provide a database for modeling hypersonic entry in a partially ionized gas under non-equilibrium, the electron-impact excitation cross sections of atoms have been calculated using perturbation theory. The energy levels covered in the calculation are retrieved from the level list in the HyperRad code. The downstream flow-field is determined by solving a set of continuity equations for each component. The individual structure of each energy level is included. These equations are then complemented by the Euler system of equations. Finally, the radiation field is modeled by solving the radiative transfer equation.
\end{abstract}

\section{Introduction}

$\mathrm{D}$ uring hypersonic entry into a planetary or lunar atmosphere, space vehicles may encounter a high temperature and/or rarified gas environment such that the gaseous flows around them could be significantly affected by various non-equilibrium phenomena. In the high temperature regime, the flow field will also become partially ionized. The percentage of ionization depends on the entry speed, vehicle size, and atmospheric composition. The electrons and atomic/molecular ions produced by the ionization introduce new reaction mechanisms that significantly influence the non-equilibrium processes and thus the radiative and convective heat loads.

\footnotetext{
${ }^{\text {a }}$ Research Scientist, Science and Technology Corp. Member AIAA, Winifred.Huo@nasa.gov

${ }^{\mathrm{b}}$ Research Scientist, Member AIAA, Yen.liu@nasa.gov

c Assistant Professor, Member AIAA, mpanesi@illinois.edu.

d Postdoctoral Research Associate, munafo@illinois.edu.

e Research Scientist, alan.wray@nasa.gov

${ }^{\mathrm{f}}$ Research Scientist, duane.f.carbon@nasa.gov
} 
In this regime, electron-atom/molecule collisions provide an efficient means of populating excited electronic states of the atoms and molecules in the flow field. Thus, collisions play an important role in determining the internal energy and state distribution of the gaseous particles. The excited states in turn are the source of radiation occurring during a hypersonic entry. Electron-atom/molecule collisions differ from heavy particle collisions in two aspects. First, the mass of an electron is more than four orders of magnitude smaller than the reduced mass of $\mathrm{N}_{2}$. Thus its average speed, and hence its average collision frequency, is more than 100 times larger. Even in the slightly ionized regime with only $1 \%$ electrons, the frequency of electron-atom/molecule collisions is equal to or larger than that of heavy particle collisions, an important consideration in the low density part of the atmosphere where the reaction probability is frequently controlled by the collision frequency. Second, the interaction potential between a charged particle (electron) and a neutral particle is longer range than neutral-neutral interactions. Hence electron-atom/molecule collision cross sections tend to be larger. Another characteristic of electron-heavy particle collisions is that they generally produce a variety of excited-state species whereas heavy particle collisions tend to produce specific excited states. Also, low-energy electron collisions can be effective in spin-changing excitations.

A one-dimensional flow solver coupled to the gas kinetics and to radiative transfer is used to illustrate how ionization and electron collisions influence flow properties and radiative and convective heat loads. One important aspect in this study is the explicit coupling of the species conservation equations with radiation by solving the radiative transfer equation, instead of employing an escape factor to approximately account for the effect of the radiative processes on the populations of the excited states. ${ }^{1,2}$ This results in a self-consistent modeling of the radiative processes. Two important issues for re-entry applications into the Earth's atmosphere are considered: (1) the treatment of the time-dependent chemistry of an ionizing air plasma for different re-entry conditions, and (2) the importance of a consistent treatment of kinetics and radiation, via the numerical solution of the radiative transport equation coupled with the set of kinetic equations.

\section{Electron-impact excitation of atoms}

Modeling electron collisions in non-equilibrium gas dynamics requires data to simulate their production, removal, and energy transfer in the flow field. Using a combination of experimental data and a variety of approximate formulas, several databases have been developed by the entry physics community. The data sets by Park $^{3,4}$ and Losev, ${ }^{5}$ and Bird's TCE ${ }^{6,7}$ models are well-established examples of this approach. The NEQAIR package $^{8}$ that simulates non-equilibrium radiation in an entry flow employs Gryzinski's classical formula ${ }^{9}$ for electron-impact excitation of atoms, whereas for molecules experimental data are used, sometimes by extrapolation or by analogy. More recent models have incorporated improved databases, based on new experimental data and/or theoretical calculations. The ABBA model by Bultel et al. ${ }^{10}$ uses the semi-empirical Drawin formula ${ }^{11,12}$ but also has incorporated many updates. Similarly SPRADIAN $07^{13}$ incorporates new, improved data into the NEQAIR model.

Due to the light electron mass, in general e-atom ${ }^{\mathrm{g}}$ collisions should be treated using quantum mechanics instead of classical mechanics. To date, only limited quantal data have been used in gas dynamics calculations. In a quantum treatment, the Schrödinger equation for an e-atom system is given by

$$
\left(\begin{array}{ll}
H & E
\end{array}\right) \quad\left({ }_{1} \cdots_{n+1}, \mathbf{R}_{1} \cdots \mathbf{R}_{M}\right)=0 .
$$

Here $H$ is the Hamiltonian of the e + target system, $E$ the total energy, $\Psi$ the corresponding wave function, $\tau_{i}$ the spatial $\left(\mathbf{r}_{i}\right)$ and spin $\left(\mathbf{s}_{i}\right)$ coordinates of the $i^{\text {th }}$ electron, and $\mathbf{R}_{K}$ the spatial coordinate of the $K^{\text {th }}$ nucleus. In what follows, the indices $i$ and $j$ label the $n$ bound electrons, and $n+l$ labels the free electron. Due to the large difference between electron and nuclear masses, it is customary to use the center of mass of the target as the center of mass of the colliding system and ignore nuclear motion. The total Hamiltonian consists of the target Hamiltonian, $H_{A}$, the kinetic energy operator of the free electron, $\mathcal{T}_{e}$, and the Coulomb potential $V$ between the free electron and the target.

$$
H=H_{A}+\mathcal{T}_{e}+V
$$

g The term "atom" refers to the heavy particle target. The result is also applicable to molecules. 


$$
\begin{gathered}
H_{A}=\frac{1}{2} \sum_{i=1}^{n} \nabla_{i}^{2} \frac{1}{2} \sum_{K=1}^{M} \nabla_{K}^{2}+\sum_{j>i}^{n} \sum_{i=1}^{n} \frac{1}{\left|\mathbf{r}_{j} \quad \mathbf{r}_{i}\right|} \sum_{K=1}^{M} \sum_{i=1}^{n} \frac{Z_{K}}{\left|\mathbf{r}_{i} \quad \mathbf{R}_{K}\right|}+\sum_{L>K}^{M} \sum_{K=1}^{M} \frac{Z_{K} Z_{L}}{\left|\mathbf{R}_{L} \mathbf{R}_{K}\right|}, \\
\mathcal{T}_{e}=\frac{1}{2} \nabla_{n+1}^{2}, \\
V={ }_{i}^{n} \frac{1}{\left|\mathbf{r}_{n+1} \mathbf{r}_{i}\right|}{ }_{K=1}^{M} \frac{Z_{K}}{\left|\mathbf{r}_{n+1} \mathbf{R}_{K}\right|} .
\end{gathered}
$$

Here $Z_{K}$ is the charge of the $K^{\text {th }}$ nucleus.

To generate a database of electron-impact excitation cross sections, we seek the solution of Eq. (1) for all states of the atom. (In the present study, the list of states are truncated and are taken from the level list in the HyperRad code ${ }^{14}$.) Generally, two methods are in common use for such calculations: the variational method and the perturbation method. The variational method seeks the solution based on optimizing the functions among a set of variational parameters. It is effective in treating strong coupling among target states and resonances and has been frequently applied to low-energy electron collisions. However, it has the limitation of treating only 30 or so target states. In the perturbation method the scattering wave function in Eq. (1) is expressed as ${ }^{15}$

$$
={ }_{0}+G^{(+)} V
$$

Here $\Phi_{0}$ is the eigenfunction of the non-interacting Hamiltonian $H_{A}+\mathcal{T}_{e}$, and the Green's function $G^{(+)}$is given by

$$
G^{(+)}=\left(\begin{array}{ll}
E & H+i
\end{array}\right)^{1}
$$

with $\delta$ a vanishingly small positive number. The Green's function $G_{0}^{(+)}$of the non-interacting Hamiltonian is given by

$$
G_{0}^{(+)}=\left(\begin{array}{lll}
E & H_{A} & \mathcal{T}_{e}+i
\end{array}\right)^{1}
$$

The Green's function $G^{(+)}$can be expressed in terms $G_{0}^{(+)}$,

$$
G^{(+)}=G_{0}^{(+)}+G_{0}^{(+)} V G^{(+)}=G_{0}^{(+)}+G_{0}^{(+)} V G_{0}^{(+)}+G_{0}^{(+)} V G_{0}^{(+)} V G_{0}^{(+)}+\cdots
$$

The perturbation expansion of the scattering wave function $\Psi$ is obtained by substituting Eq. (9) into (6).

$$
\Psi=\Phi_{0}+G_{0}^{(+)} V \Phi_{0}+G_{0}^{(+)} V G_{0}^{(+)} V \Phi_{0}+G_{0}^{(+)} V G_{0}^{(+)} V G_{0}^{(+)} V \Phi_{0} \cdots
$$

Since $\Phi_{0}$ is the product of the atomic wave function and a plane wave that describes the free electron, it is easier to calculate. A first order perturbation treatment uses only the first term in Eq. (10), a second order treatment uses two terms, etc. Perturbation treatments work best when the coupling among atomic states during the collision process is weak and resonance is unimportant, e.g., in high-energy collisions. However, some approximate formulas also seem to work quite well in low-energy collisions. A major advantage of the perturbation treatment lies in the fact that it allows the calculation of electron collisions with the full set of atomic states, instead of just a limited number.

The atomic level list for $\mathrm{N}, \mathrm{O}$, and $\mathrm{C}$ atoms in the HyperRad code, ${ }^{14}$ compiled by merging the NIST, ${ }^{16}$ Vanderbilt ${ }^{17}$ and TOPBase ${ }^{18}$ databases, consists of 684 states of N, 552 states of O, and 915 states of C. ${ }^{19}$ In view of the number of atomic states involved, the variational method cannot be employed to handle all the transitions. Thus the perturbation treatment is used to calculate the electron-impact cross sections. For N, O, and C, electronimpact excitation cross sections from the ground and the first two metastable states to selected excited states have 
been calculated using the B-spline R-matrix method. ${ }^{20,21,22,23}$ The excitation rate coefficients of $\mathrm{O}$ and $\mathrm{C}$ from the B-spline R-matrix calculations have also been tabulated. ${ }^{23,24}$ For $\mathrm{N}$ the rate coefficients calculated using the conventional R-matrix method are available. ${ }^{25}$ In total there are R-matrix excitation rate coefficients for 57 transitions in N, 53 in $\mathrm{O}$, and 66 in C. Note, for example, the total cross section and rate coefficients for N, based on the NIST-Vanderbilt-TOPBase data in HyperRad, consists of 467,172 transitions. Thus the R-matrix data is far from sufficient. The R-matrix cross sections and rate coefficients are used to supplement our dataset. They are also used, together with experimental measurements, to benchmark our calculations based on the perturbation method.

In the perturbation calculation, the excitation processes can be grouped according to the interaction potential responsible for the transition. By expanding the interaction potential in Eq. (5) in terms of multipole moments and grouping the excitation process according to the multipole moment responsible for the transition, we have the following processes for electron-neutral atom excitations

- Dipole transitions

- Quadrupole transitions

- Magnetic dipole transitions

- Symmetry-forbidden transitions

- Exchange scattering

The dipole potential is the longest range. The incoming electron need not penetrate the atomic charge cloud for such transitions to occur. The quadrupole and magnetic dipole potentials are shorter range. The shortest range potential is for symmetry-forbidden transitions. Their cross sections tend to be smaller than dipole-allowed transitions. Exchange scattering is based on a different mechanism wherein the free electron becomes bound after collision and an originally bound electron becomes free. While the exchange mechanism applies to all transitions, it is the dominant mechanism for spin-forbidden transitions. For the atoms under consideration, the dipole-allowed transitions generally have the largest cross sections. On the other hand, the quadrupole, magnetic dipole, and symmetry-forbidden transition cross sections are smaller than the exchange contribution. Thus, in the following only dipole-allowed and exchange mechanisms are considered.

\section{A. Dipole allowed excitation}

The differential cross section in first order perturbation theory (also called the Born cross section) is given by

$$
\begin{gathered}
\frac{d \sigma_{f o}}{d \Omega}=\frac{1}{(2 \pi)^{2}} \frac{k_{f}}{k_{o}}\left|\int \exp \left(i \vec{K} \bullet \vec{r}_{n+1}\right) \phi_{f}^{*}\left(\vec{r}_{1} \cdots \vec{r}_{n}\right) V \phi_{o}\left(\vec{r}_{1} \cdots \vec{r}_{n}\right) d \vec{r}_{1} \cdots d \vec{r}_{n} d \vec{r}_{n+1}\right|^{2}, \\
\vec{K}=\vec{k}_{o} \quad \vec{k}_{f}
\end{gathered}
$$

The subscripts $o$ and $f$ denote the initial and final states of the collision, $\vec{k}_{o}$ and $\vec{k}_{f}$ are the initial and final momenta of the free electron, $\vec{K}$ is the momentum transfer, and $\phi_{o}$ and $\phi_{f}$ are the initial and final wavefunctions of the target. A related quantity, the generalized oscillator strength, $f(K)$, is expressed as

$$
f(K)=\frac{2 W}{K^{2}}\left|\left\langle\phi_{f}\left|\sum_{i=1}^{n} \exp \left(i \vec{K} \cdot \vec{r}_{i}\right)\right| \phi_{o}\right\rangle\right|^{2},
$$

where $\mathrm{W}$ is the excitation energy for the ${ }_{o} \rightarrow f_{f}$ transition of the target. In the Born approximation, the generalized oscillator strength for dipole-allowed transitions is given by ${ }^{26}$

$$
f=\frac{1}{\left(1+x^{2}\right)^{6}} f_{o}+e_{n=1} \frac{x}{n}^{n}
$$

where $f_{o}$ is the optical oscillator strength and 


$$
\begin{aligned}
& x=\frac{K}{a}, \\
& a=\sqrt{2 I}+\sqrt{2(I \quad W),}
\end{aligned}
$$

where $I$ is the ionization potential of the initial target state. The generalized oscillator strength in Eq. (14) satisfies both Lassettre's limit theorem ${ }^{27}$ at $K=0$ and the asymptotic form of Rau and Fano ${ }^{28}$ at large $K$.

The integral cross section is given by

$$
\begin{aligned}
f_{o} & =\frac{2}{W k_{o}^{2}} \quad K_{\min }^{2} \frac{f\left(K^{2}\right)}{K^{2}} d K^{2} . \\
K_{\min }^{2} & =k_{o}^{2}+k_{f}^{2} \quad 2 k_{o} k_{f}, \\
K_{\max }^{2} & =k_{o}^{2}+k_{f}^{2}+2 k_{o} k_{f} .
\end{aligned}
$$

Using the first term in the series expansion in Eq. (9), the one-term Born cross section is given by

$$
f_{o}=\frac{2}{W} \frac{f_{o}}{k_{o}^{2}}\left|\frac{1}{5(1+x)^{5}}+\frac{1}{4(1+x)^{4}}+\frac{1}{3(1+x)^{3}}+\frac{1}{2(1+x)^{2}}+\frac{1}{(1+x)} \ln \frac{1+x}{x} \div\right|_{K_{\min }^{2} / a^{2}}^{K_{\max }^{2} / a^{2}} .
$$

Two other perturbation treatments that include higher order corrections are also considered: the BE-scaling method of $\mathrm{Kim}^{29}$ and the modified Born cross section in Eq. (29) in the paper by Dillon and Lassettre ${ }^{26}$. In the electron energy range of interest in hypersonic entry, threshold to $30 \mathrm{eV}$, the one-term Born formula is found to perform better than the BE-scaling method and the modified Born cross section. Thus, the one-term Born formula is used.

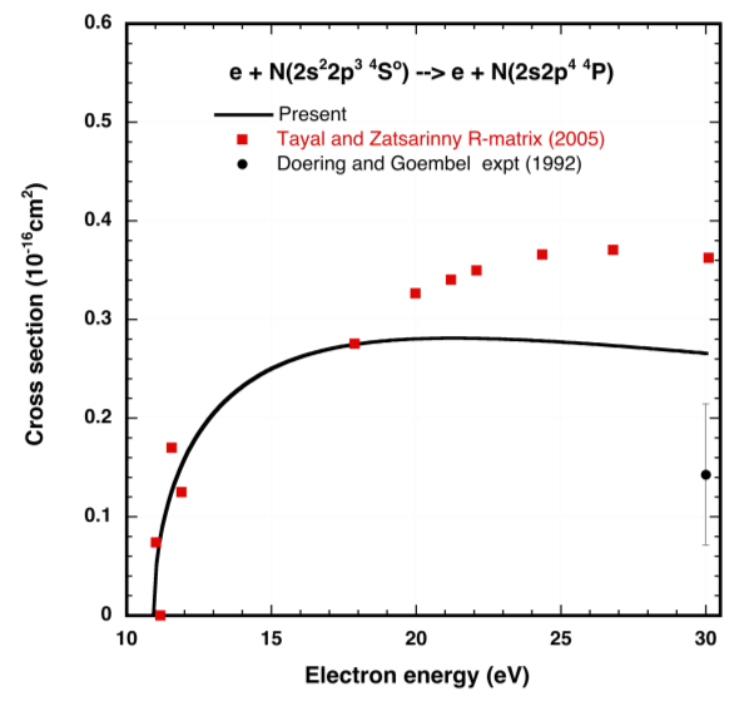

(a)

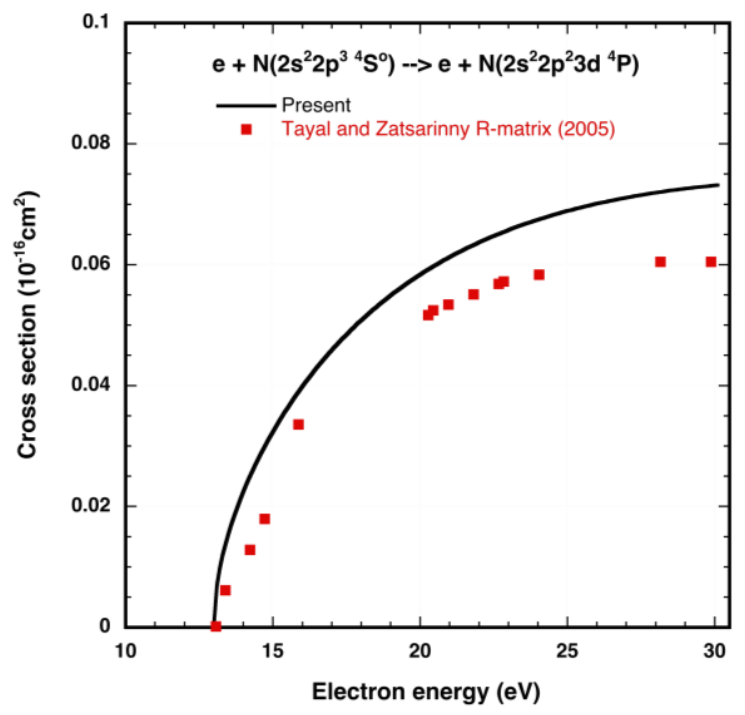

(b)

Figure 1. Electron impact excitation cross sections of $N$ atom. (a) $2 s^{2} 2 p^{3}{ }^{4} S^{o} \rightarrow 2 s 2 p^{4} P$. (b) $2 s^{2} 2 p^{3}{ }^{4} S^{\circ} \rightarrow 2 s^{2} 2 p^{2}\left({ }^{3} \mathrm{P}\right) 3 d{ }^{4} \mathrm{P}$. Black curve, present perturbation calculation. Red squares, Bspline R-matrix calculation of Tayal and Zatsarinny ${ }^{20}$. Black dot, experimental data by Doering and Goembel ${ }^{30}$. 


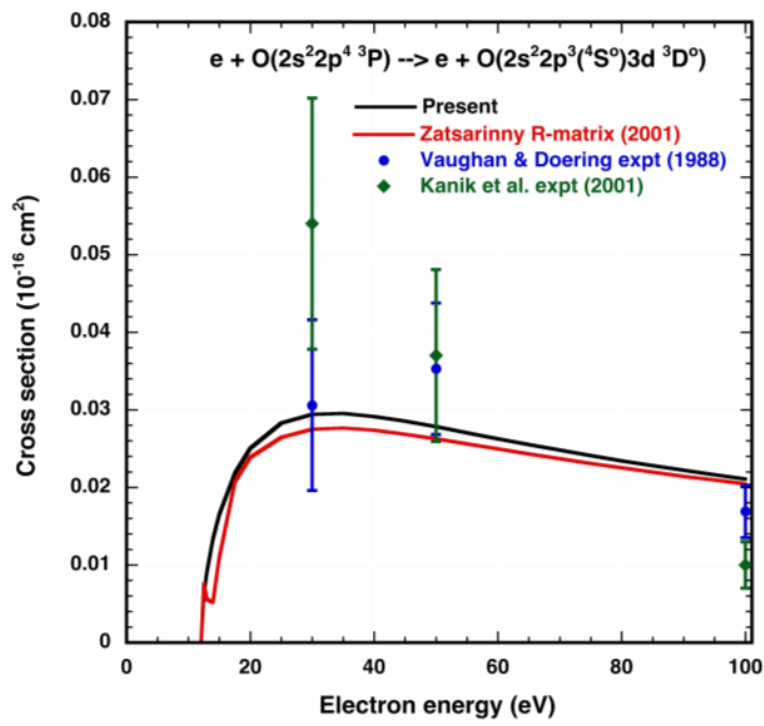

(a)

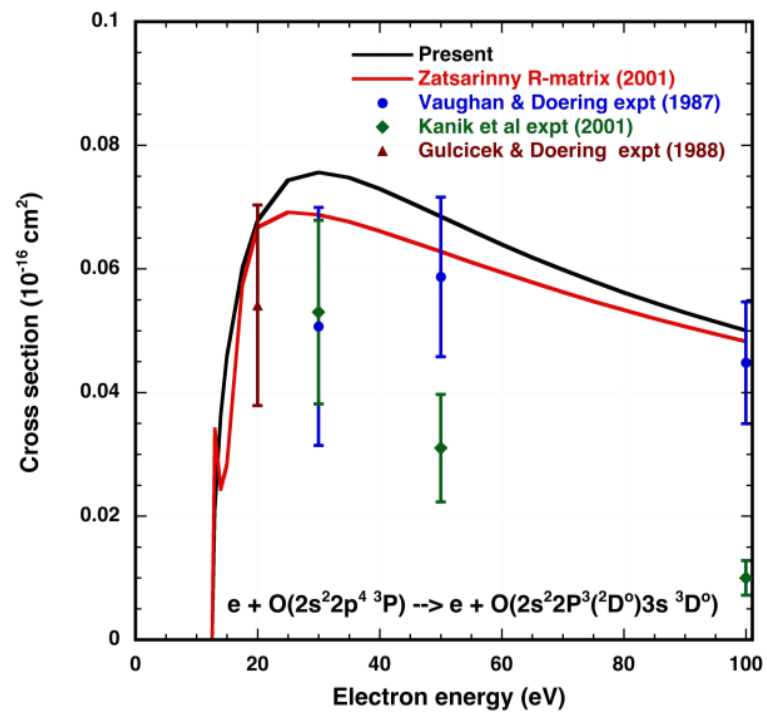

(b)

Figure 2. Electron impact excitation cross sections of $\mathrm{O}$ atom. (a) $2 \mathrm{~s}^{2} 2 \mathrm{p}^{4}{ }^{3} \mathrm{P} \rightarrow 2 \mathrm{~s}^{2} 2 \mathrm{p}^{3}\left({ }^{4} \mathrm{~S}^{\mathrm{o}}\right) 3 \mathrm{~d}^{3} \mathrm{D}^{\mathrm{o}}$. (b) $2 \mathrm{~s}^{2} 2 \mathrm{p}^{4}{ }^{3} \mathrm{P} \rightarrow 2 \mathrm{~s}^{2} 2 \mathrm{p}^{3}\left({ }^{2} \mathrm{D}^{\mathrm{o}}\right) 3 \mathrm{~s}^{3} \mathrm{D}^{\circ}$. Black curve, present perturbation calculation. Red curve, Bspline R-matrix calculation of Tayal and Zatsarinny ${ }^{22}$. For the $2 \mathrm{~s}^{2} 2 \mathrm{p}^{4}{ }^{3} \mathrm{P} \rightarrow 2 \mathrm{~s}^{2} 2 \mathrm{p}^{3}\left({ }^{4} \mathrm{~S}^{\mathrm{o}}\right) 3 \mathrm{~d}^{3} \mathrm{D}^{\mathrm{o}}$ transition, blue dots, experiment data of Vanghan $\&$ Doering ${ }^{31}$, green diamonds, Kanik et al ${ }^{32}$. For the $2 \mathrm{~s}^{2} 2 \mathrm{p}^{4}{ }^{3} \mathrm{P} \rightarrow 2 \mathrm{~s}^{2} 2 \mathrm{p}^{3}\left({ }^{2} \mathrm{D}^{\mathrm{o}}\right) 3 \mathrm{~s}{ }^{3} \mathrm{D}^{\circ}$ transition, blue dots, experimental data of Vanghan and Doering $^{33}$, green diamonds, Kanik et $\mathrm{al}^{32}$, and red triangles, Gulcicek and Doering ${ }^{34}$.

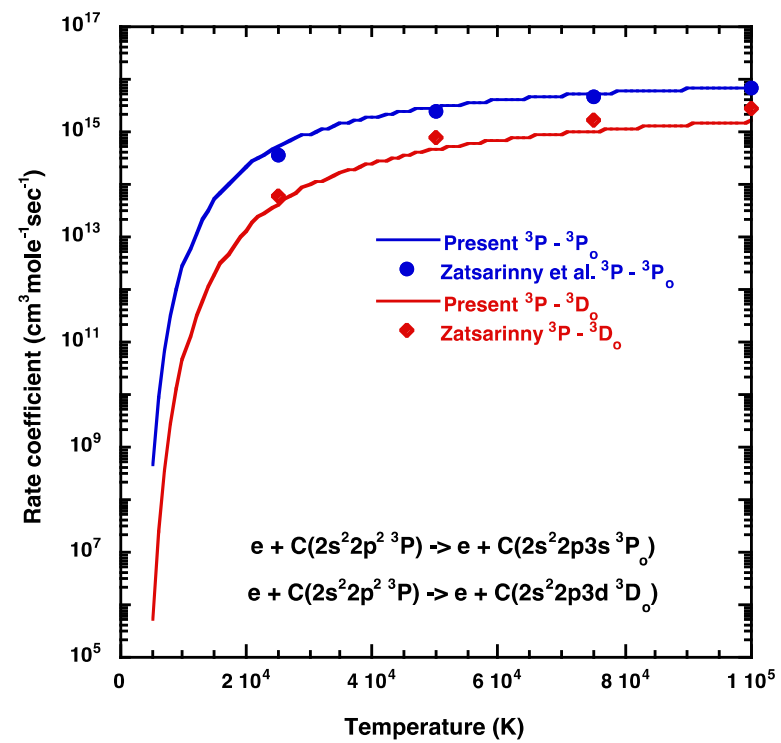

Figure 3. Electron impact excitation rate coefficients of $\mathrm{C}$ atom. Blue curve, present perturbation calculation of $2 \mathrm{~s}^{2} 2 \mathrm{p}^{2}{ }^{3} \mathrm{P} \rightarrow 2 \mathrm{~s}^{2} 2 \mathrm{p}\left({ }^{2} \mathrm{P}^{\mathrm{o}}\right) 3 \mathrm{~s}{ }^{3} \mathrm{P}^{\mathrm{o}}$. Blue circles, B-spline R-matrix calculation of Zatsarinny et al. ${ }^{35}$ for this transition. Red curve, present calculation of $2 \mathrm{~s}^{2} 2 \mathrm{p}^{2}{ }^{3} \mathrm{P} \rightarrow 2 \mathrm{~s}^{2} 2 \mathrm{p}\left({ }^{2} \mathrm{P}^{\mathrm{o}}\right) 3 \mathrm{~d}$ ${ }^{3} \mathrm{D}^{\mathrm{o}}$. Blue diamonds, B-spline R-matrix calculation of Zatsarinny et al. for the same transition. 
Figure 1 compares the electron-impact excitation cross sections from the present perturbation calculation for $\mathrm{N}$ atom with the B-spline R-matrix with Pseudo States calculation of Tayal and Zatsarinny ${ }^{20}$ using 24 spectroscopic bound and auto-ionizing states together with 15 pseudo states. Fig. 1(a) shows the results for $2 s^{2} 2 p^{3}{ }^{4} S^{o} \rightarrow 2 s 2 p^{4} P$ of $\mathrm{N}$ and Fig. 1(b) the results for the $2 \mathrm{~s}^{2} 2 \mathrm{p}^{3}{ }^{4} \mathrm{~S}^{\mathrm{o}} \rightarrow 2 \mathrm{~s}^{2} 2 \mathrm{p}^{2}\left({ }^{3} \mathrm{P}\right) 3 \mathrm{~d}^{4} \mathrm{P}$ of $\mathrm{N}$. For $\mathrm{N} 2 \mathrm{~s}^{2} 2 \mathrm{p}^{3}{ }^{4} \mathrm{~S}^{\mathrm{o}}-2 \mathrm{~s} 2 \mathrm{p}^{4}{ }^{4} \mathrm{P}$, the single experimental data at $30 \mathrm{eV}$ is by Doering and Goembel ${ }^{30}$. Figure 2 compares the present calculation for $\mathrm{O}$ atom with the B-spline R-matrix calculations of Zatsarinny and Tayal ${ }^{22}$ using 26 spectroscopic and autoionizing states. Figure 2(a) shows the results for $2 s^{2} 2 p^{4}{ }^{3} \mathrm{P} \rightarrow 2 \mathrm{~s}^{2} 2 \mathrm{p}^{3}\left({ }^{4} \mathrm{~S}^{\circ}\right) 3 \mathrm{~d}^{3} \mathrm{D}^{\circ}$ of $\mathrm{O}$ and Figure 2(b) shows the results for $2 \mathrm{~s}^{2} 2 \mathrm{p}^{4}{ }^{3} \mathrm{P}$ $\rightarrow 2 \mathrm{~s}^{2} 2 \mathrm{p}^{3}\left({ }^{2} \mathrm{D}^{\circ}\right) 3 \mathrm{~s}^{3} \mathrm{D}^{\mathrm{o}}$ of O. Comparisons are also made with the experimental data of Vaughan and Doering ${ }^{31,33}$, Kanik et al. ${ }^{32}$, and Gulcicek and Doering ${ }^{34}$. Figure 3 compares the electron-impact excitation rate coefficients of $\mathrm{C}$ atom from the present calculation with the B-spline R-matrix calculation of Zatsarinny et al. ${ }^{35}$ using 28 spectroscopic states and 8 pseudo states. The two transition presented are $2 s^{2} 2 p^{2}{ }^{3} \mathrm{P} \rightarrow 2 \mathrm{~s}^{2} 2 \mathrm{p}\left({ }^{2} \mathrm{P}^{\mathrm{o}}\right) 3 \mathrm{~s}^{3} \mathrm{P}^{\mathrm{o}}$ and $2 \mathrm{~s}^{2} 2 \mathrm{p}^{2}$ ${ }^{3} \mathrm{P} \rightarrow 2 \mathrm{~s}^{2} 2 \mathrm{p}\left({ }^{2} \mathrm{P}^{\circ}\right) 3 \mathrm{~d}^{3} \mathrm{D}^{\circ}$. No experimental data are available for e-C excitations.

Figure 4 shows electron-impact excitation rate coefficients for $\mathrm{N}$ atom from the present calculation as a function of temperature, for selected states from the present calculation.

(1). $2 \mathrm{~s}^{2} 2 \mathrm{p}^{3}{ }^{4} \mathrm{~S}^{\circ} J=3 / 2 \rightarrow 2 \mathrm{~s}^{2} 2 \mathrm{p}^{2}\left({ }^{3} \mathrm{P}\right) 9 \mathrm{~s}{ }^{4} \mathrm{P} J=5 / 2, \mathrm{~W}=115,576.59 \mathrm{~cm}^{-1}$.

(2). $2 \mathrm{~s}^{2} 2 \mathrm{p}^{3}{ }^{4} \mathrm{~S}^{\circ} J=3 / 2 \rightarrow 2 \mathrm{~s}^{2} 2 \mathrm{p}^{2}\left({ }^{3} \mathrm{P}\right) 4 \mathrm{~d}^{4} \mathrm{P} J=3 / 2, \mathrm{~W}=110,322.72 \mathrm{~cm}^{-1}$.

(3). $2 \mathrm{~s}^{2} 2 \mathrm{p}^{3}{ }^{2} \mathrm{D}^{\mathrm{o}} J=5 / 2 \rightarrow 2 \mathrm{~s}^{2} 2 \mathrm{p}^{2}\left({ }^{3} \mathrm{P}\right) 4 \mathrm{~d}^{2} \mathrm{~F} J=7 / 2, \mathrm{~W}=91,138.0 \mathrm{~cm}^{-1}$.

(4) $2 \mathrm{~s}^{2} 2 \mathrm{p}^{3}{ }^{2} \mathrm{D}^{\circ} J=3 / 2 \rightarrow 2 \mathrm{~s}^{2} 2 \mathrm{p}^{2}\left({ }^{3} \mathrm{P}\right) 3 \mathrm{~d}^{2} \mathrm{P} J=1 / 2, \mathrm{~W}=85,420.85 \mathrm{~cm}^{-1}$.

(5) $2 \mathrm{~s}^{2} 2 \mathrm{p}^{3}{ }^{2} \mathrm{P}^{\mathrm{o}} J=3 / 2 \rightarrow 2 \mathrm{~s}^{2} 2 \mathrm{p}^{2}\left({ }^{3} \mathrm{P}\right) 4 \mathrm{~d}^{2} \mathrm{P} J=1 / 2, \mathrm{~W}=81,405.88 \mathrm{~cm}^{-1}$.

(6) $2 \mathrm{~s}^{2} 2 \mathrm{p}^{2}\left({ }^{3} \mathrm{P}\right) 3 \mathrm{p}{ }^{4} \mathrm{D}^{\mathrm{o}} J=3 / 2 \rightarrow 2 \mathrm{~s}^{2} 2 \mathrm{p}^{2}\left({ }^{3} \mathrm{P}\right) 5 \mathrm{~d}^{4} \mathrm{D} J=5 / 2, \mathrm{~W}=18,117.14 \mathrm{~cm}^{-1}$.

(7) $2 \mathrm{~s}^{2} 2 \mathrm{p}^{2}\left({ }^{3} \mathrm{P}\right) 3 \mathrm{p}{ }^{4} \mathrm{P}^{\mathrm{o}} J=5 / 2 \rightarrow 2 \mathrm{~s}^{2} 2 \mathrm{p}^{2}\left({ }^{3} \mathrm{P}\right) 5 \mathrm{~s}{ }^{4} \mathrm{P} J=3 / 2, \mathrm{~W}=14,324.37 \mathrm{~cm}^{-1}$.

(8) $2 \mathrm{~s}^{2} 2 \mathrm{p}^{2}\left({ }^{3} \mathrm{P}\right) 3 \mathrm{p}{ }^{4} \mathrm{D}^{\mathrm{o}} J=3 / 2 \rightarrow 2 \mathrm{~s}^{2} 2 \mathrm{p}^{2}\left({ }^{3} \mathrm{P}\right) 3 \mathrm{~d}{ }^{2} \mathrm{~F} J=5 / 2, \mathrm{~W}=9,979.47 \mathrm{~cm}^{-1}$.

(9) $2 \mathrm{~s}^{2} 2 \mathrm{p}^{2}\left({ }^{1} \mathrm{D}\right) 3 \mathrm{~s}^{2} \mathrm{D} J=3 / 2 \rightarrow 2 \mathrm{~s}^{2} 2 \mathrm{p}^{2}\left({ }^{3} \mathrm{P}\right) 4 \mathrm{p}^{2} \mathrm{P}^{\mathrm{o}} J=1 / 2, \mathrm{~W}=7,924.56 \mathrm{~cm}^{-1}$.

(10) $2 \mathrm{~s}^{2} 2 \mathrm{p}^{2}\left({ }^{3} \mathrm{P}\right) 3 \mathrm{~d}^{4} \mathrm{~F} J=5 / 2 \rightarrow 2 \mathrm{~s}^{2} 2 \mathrm{p}^{2}\left({ }^{3} \mathrm{P}\right) 5 \mathrm{p}{ }^{4} \mathrm{D}^{\mathrm{o}} J=5 / 2, \mathrm{~W}=6,520.96 \mathrm{~cm}^{-1}$.

(11) $2 \mathrm{~s}^{2} 2 \mathrm{p}^{2}\left({ }^{3} \mathrm{P}\right) 5 \mathrm{p}^{4} \mathrm{D}^{\mathrm{o}} J=1 / 2 \rightarrow 2 \mathrm{~s}^{2} 2 \mathrm{p}^{2}\left({ }^{1} \mathrm{~S}\right) 3 \mathrm{~s}^{2} \mathrm{~S} J=1 / 2, \mathrm{~W}=5,079.71 \mathrm{~cm}^{-1}$.

(12) $2 \mathrm{~s}^{2} 2 \mathrm{p}^{2}\left({ }^{3} \mathrm{P}\right) 3 \mathrm{~d}^{2} \mathrm{P} J=1 / 2 \rightarrow 2 \mathrm{~s}^{2} 2 \mathrm{p}^{2}\left({ }^{3} \mathrm{P}\right) 4 \mathrm{p}^{2} \mathrm{P}^{\mathrm{o}} J=1 / 2, \mathrm{~W}=2,934.44 \mathrm{~cm}^{-1}$.

(13) $2 \mathrm{~s}^{2} 2 \mathrm{p}^{2}\left({ }^{3} \mathrm{P}\right) 3 \mathrm{~d}{ }^{4} \mathrm{P} J=3 / 2 \rightarrow 2 \mathrm{~s}^{2} 2 \mathrm{p}^{2}\left({ }^{3} \mathrm{P}\right) 4 \mathrm{p}^{4} \mathrm{D}^{\mathrm{o}} J=5 / 2, \mathrm{~W}=1,954.73 \mathrm{~cm}^{-1}$.

(14) $2 \mathrm{~s}^{2} 2 \mathrm{p}^{2}\left({ }^{3} \mathrm{P}\right) 5 \mathrm{~s}{ }^{4} \mathrm{P} J=3 / 2 \rightarrow 2 \mathrm{~s}^{2} 2 \mathrm{p}^{2}\left({ }^{3} \mathrm{P}\right) 5 \mathrm{p}^{4} \mathrm{D}^{\mathrm{o}} J=5 / 2, \mathrm{~W}=1,347.5 \mathrm{~cm}^{-1}$.

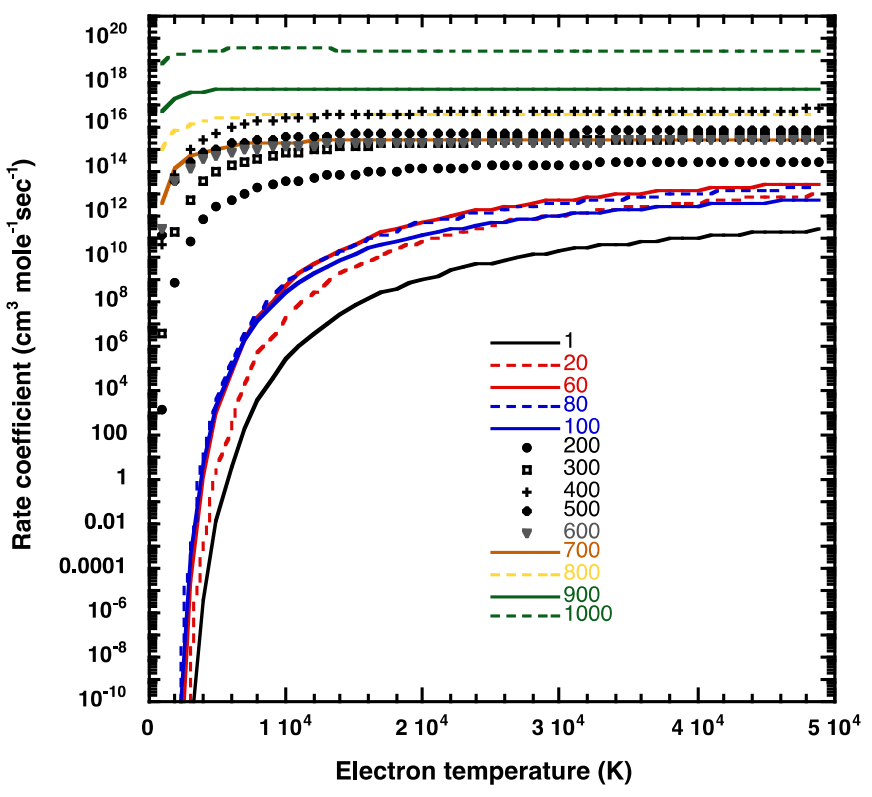

Figure 4. Electron-impact excitation rate coefficients for selected transitions in $\mathrm{N}$ atom. 
As expected, the magnitudes of the rate coefficients are influenced both by the excitation cross section and the excitation energy. Note that the energy levels of $\mathrm{N}$ atom in the calculation of the rate coefficients in Fig. 4 obey the intermediate angular momentum coupling scheme as designated in the NIST database ${ }^{16}$. Thus, the calculated rate coefficients are directly linked to radiative transitions and can be readily employed in a non-equilibrium radiation calculation. In contrast, LS coupling is used in the calculations in Figs. 1-3 in order to compare with the R-matrix results.

\section{B. Exchange scattering}

In first order perturbation theory, the differential cross section for exchange scattering is given by

$$
\begin{aligned}
& \frac{d \sigma_{f o}}{d \Omega}=\frac{k_{f}}{k_{o}}\left|g_{e x}\right|^{2}, \\
& g_{e x}=\frac{1}{2 \pi} \int \exp \left(\vec{k}_{f} \bullet \vec{r}_{n+1}\right) \phi_{f}^{*}\left(\vec{r}_{1} \cdots \vec{r}_{n}\right) V \AA \exp \left(\vec{k}_{o} \bullet \vec{r}_{n+1}\right) \phi_{o}\left(\vec{r}_{1} \cdots \vec{r}_{n}\right) d \vec{r}_{1} \cdots d \vec{r}_{n} d \vec{r}_{n+1} .
\end{aligned}
$$

Here $g_{e x}$ is the exchange scattering amplitude, and the antisymmetrizer $\mathcal{A}$ permutes the free electron coordinate with the bound electron coordinate. It is expected that the exchange with the outermost bound electron dominates the scattering process. Hence we employ the active electron approximation and include only the exchange with the outermost electron in our calculation. Ochkur ${ }^{36}$ and Rudge ${ }^{37}$ introduced an additional approximation to $g_{e x}$ and simplified the exchange amplitude to

$$
g_{e x}(K)=\frac{2}{\left(k_{f}-i \sqrt{2 I}\right)^{2}} \int \exp \left(i K \cdot \vec{r}_{1}\right) \phi_{f}^{*}\left(\vec{r}_{1} \cdots \vec{r}_{n}\right) \phi_{o}\left(\vec{r}_{1} \cdots \vec{r}_{n}\right) d \vec{r}_{1} \cdots d \vec{r}_{n}
$$

For selected cases, they demonstrated that the above equation works quite well even at low energies. The integral cross section $\sigma_{f o}$ is obtained by integrating $\frac{d f_{o}}{d}$ over the allowable range of $K$. Following Kim's practice ${ }^{38}$, we introduced a BE scaling factor to $\sigma_{f o}$ to account for higher-order effects. The BE scaling that we use is similar to but not the same as Kim's. The integral exchange cross section is then given by

$$
\sigma_{f o}=\frac{16 \pi k_{o}^{2}}{\left(k_{o}^{2}+2 I+2 W\right)^{2}} \frac{1}{\left(k_{f}^{2}+2 I\right)^{2}} \int_{K_{\min }^{2}}^{K_{\max }^{2}}\left\{\int \exp \left(i K \bullet \vec{r}_{1}\right) \phi_{f}^{*}\left(\vec{r}_{1} \cdots \vec{r}_{n}\right) \phi_{o}\left(\vec{r}_{1} \cdots \vec{r}_{n}\right) d \vec{r}_{1} \cdots d \vec{r}_{n}\right\} d K^{2}
$$

The active electron in the target wave functions is described by a hydrogenic function with quantum defect correction $^{39}$. In Eq. (21) both the radial integral and the integration over $K^{2}$ are evaluated numerically. Note that due to the use of the active electron approximation, the present treatment is applicable for transitions where the initial and final states of the atom have the same core. A higher order treatment is required for processes involving core excitations.

Figure 5 compares the present exchange calculation of electron impact rate coefficients for four spin-forbidden transitions in $\mathrm{N}$ atom with the R-matrix calculation of Frost et $\mathrm{al}^{40}$. (a) $2 \mathrm{~s}^{2} 2 \mathrm{p}^{3}{ }^{4} \mathrm{~S}^{\mathrm{o}} \rightarrow 2 \mathrm{~s}^{2} 2 \mathrm{p}^{3}{ }^{2} \mathrm{D}^{\mathrm{o}}$, (b) $2 \mathrm{~s}^{2} 2 \mathrm{p}^{3}{ }^{4} \mathrm{~S}^{\mathrm{o}} \rightarrow$ $2 \mathrm{~s}^{2} 2 \mathrm{p}^{2}\left({ }^{3} \mathrm{P}\right) 3 \mathrm{~s}^{2} \mathrm{P}$, (c) $2 \mathrm{~s}^{2} 2 \mathrm{p}^{3}{ }^{2} \mathrm{D}^{\mathrm{o}} \rightarrow 2 \mathrm{~s}^{2} 2 \mathrm{p}^{2}\left({ }^{3} \mathrm{P}\right) 3 \mathrm{~s}^{4} \mathrm{P}$, and (d) $2 \mathrm{~s}^{2} 2 \mathrm{p}^{3} \mathrm{P}^{\mathrm{o}} \rightarrow 2 \mathrm{~s}^{2} 2 \mathrm{p}^{2}\left({ }^{3} \mathrm{P}\right) 3 \mathrm{~s}^{4} \mathrm{P}$.

Comparisons of the results of the perturbation calculations with R-matrix data and available experimental data indicate that the perturbation method in the present formulation can be applied to the energy range of interest in flow-field modeling and can fill-in the large data gap not covered by previous R-matrix calculations. This has now been done. 


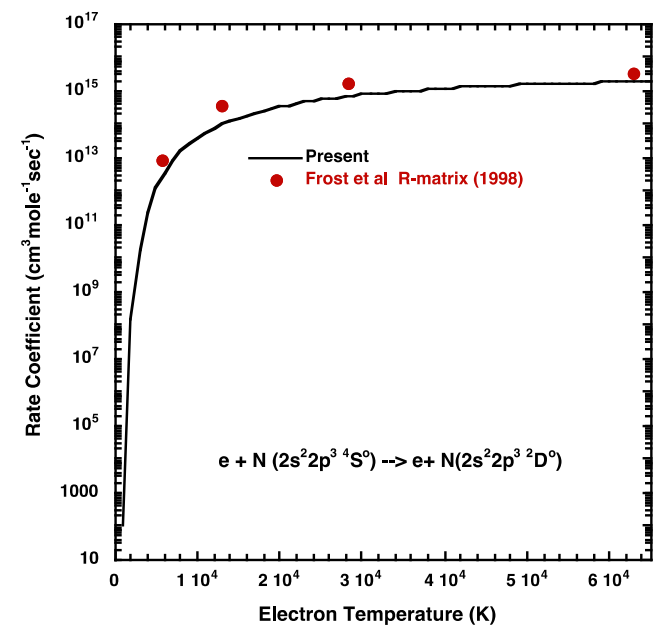

(a)

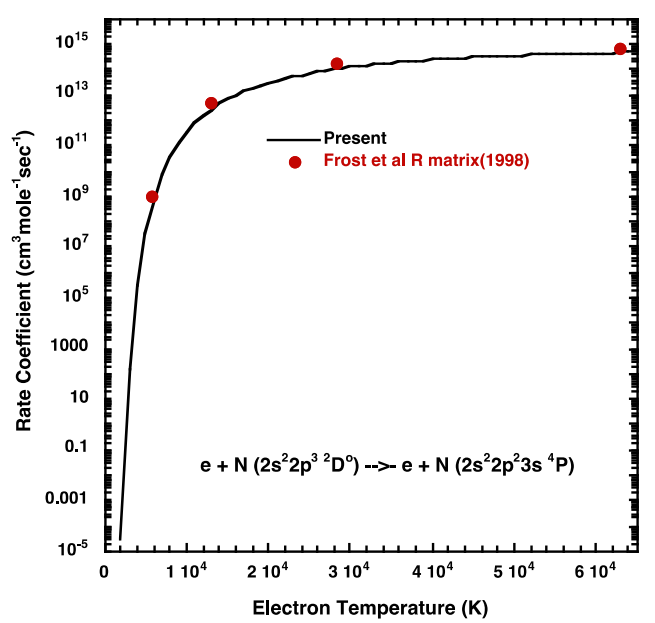

(c)

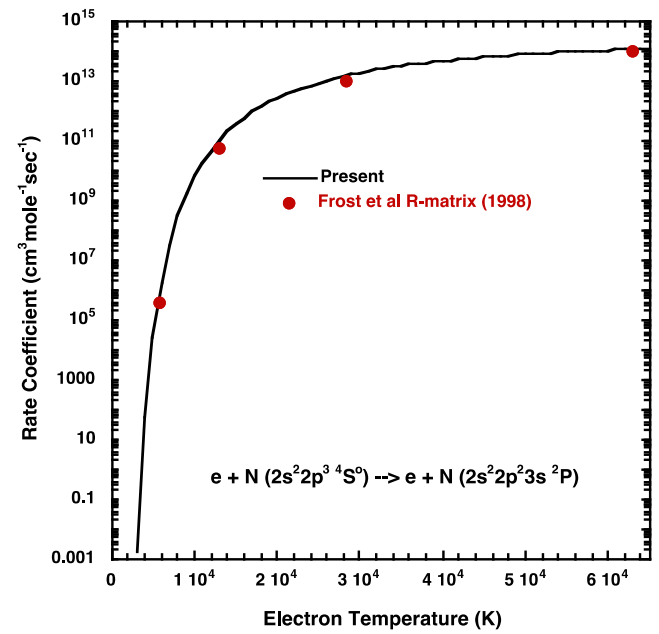

(b)

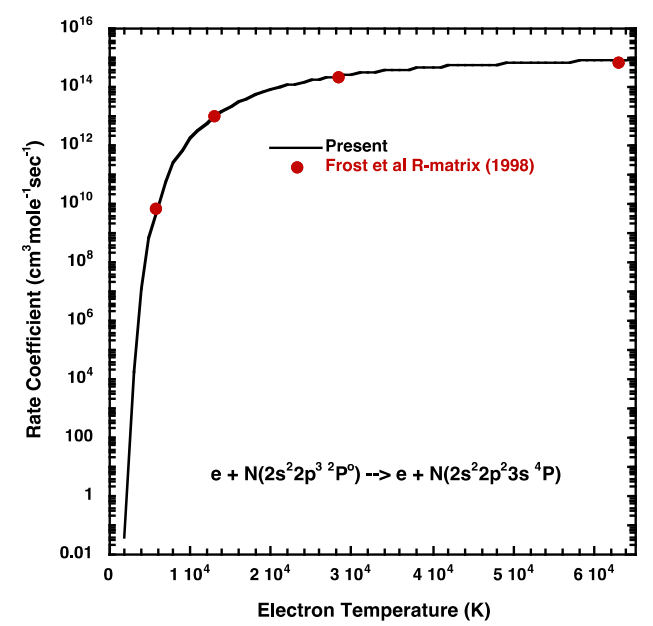

(d)

Figure 5. Electron impact excitation rate coefficients for four spin-forbidden transitions in $\mathrm{N}$ atom. Comparison of present perturbation calculation with the R-matrix calculation of Frost et $\mathrm{al}^{40}$.

\section{Numerical Results}

Previously, the HyperRad electron Stark-broadening and non-equilibrium electron-impact rate coefficient databases were based on semi-classical calculations for dipole-allowed transitions ${ }^{41}$ and Gryzinski's ${ }^{9}$ and Drawin's ${ }^{11,12}$ classical formulas for other transitions. We are currently reconstructing these databases for the electron-impact excitation of $\mathrm{N}, \mathrm{O}$, and $\mathrm{C}$ atoms using newly improved, quantal calculations. So far, we have carried out calculations of electron-impact cross-sections and rate coefficients for all dipole-allowed excitations in the HyperRad line-list database. These include 16,239 transitions for the $\mathrm{N}$ atom, 11,072 transitions for the $\mathrm{O}$ atom, and 22,546 transitions for the $\mathrm{C}$ atom. The quantal calculations involving other types of transitions will be completed in the near future. In addition, we have also computed both the dipole-allowed and exchange electronimpact cross-sections and rate coefficients for the $\mathrm{N}$ atom using the NIST level and line lists ${ }^{16}$. This includes 146 energy states below the first ionization potential, 1,249 dipole-allowed excitations, and 6,434 exchange excitations. In the following, we use this smaller set of energy states and rate coefficients to study the thermal and chemical non-equilibrium processes for two test cases. In these studies, the translational mode is assumed to be in equilibrium, satisfying a Maxwellian distribution in velocity. 


\section{A. Internal excitation and ionization of nitrogen atoms}

In the first test case, the newly computed state-to-state rate coefficients are used to study the internal excitation and ionization processes of nitrogen atoms in an isothermal and isochoric heat bath. Nitrogen atoms, seeded with a small number of nitrogen ions and electrons, are suddenly heated, and the translational temperature of the gas is instantaneously brought to a high temperature. This drives the gas toward a highly thermal and chemical nonequilibrium condition. Here we consider the internal excitation and ionization processes due to electron-impact collisions with nitrogen atoms:

$$
\begin{aligned}
N^{i}+e & \Leftrightarrow N^{j}+e \\
N^{i}+e & \Leftrightarrow N^{+}+e+e .
\end{aligned}
$$

The superscripts $i$ and $j$ in the above equations denote generic indices for internal states of a species, here the $N$ atom. The ionization rate coefficients used to model the process described by Eq. (23) are obtained from previous quantal calculations ${ }^{42}$. Due to the lack of a complete set of rate coefficients, the ionization process is simplified to include only the ground state of the first ion $N^{+}$.

Let $n^{i}, g^{i}$, and ${ }^{i}$ denote the number density, degeneracy, and energy level for energy state $i$ of a species, respectively. In this study, we further assume that the gas has no mean speed. The microscopic governing equations for $n^{i}$ are thus reduced to the master equations. These equations are solved using the ordinary differential equation package LSODE ${ }^{43}$. Note that the species total number density $n$ can be obtained by summing of all its energy-state number densities, $n=n^{i}$. Similarly, the species total internal energy $=n^{i}{ }^{i}$. For given $n$ and , provided that the $n^{i}$ are in a Boltzmann distribution, we can define a species-electronic (or internal) temperature $T^{e l}$ which satisfies the relation

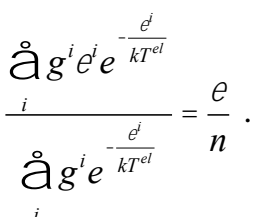

However, when the $n^{i}$ are not in a Boltzmann distribution, $T^{e l}$ has no simple physical interpretation, other than as a parameter to measure how far the gas has deviated from thermal equilibrium. One must be cautious in relating such a fictitious temperature to any physical quantities.

In the following test case, numerical calculations of $n^{i}$ are carried out assuming isothermal conditions in translational mode, with an initial temperature of $6,000 \mathrm{~K}$ and a final temperature of 30,000 K. The initial pressure is set to $100 \mathrm{~Pa}$, and the composition consists of $98 \% \mathrm{~N}, 1 \% \mathrm{~N}^{+}$, and $1 \% e^{-}$. This system has been studied previously by Liu et al. ${ }^{44}$, but the excitation and ionization rate coefficients in that work were calculated using the Drawin formula. Figures (6a) and (6b) compare the atomic nitrogen and electron mole fractions and the atomic nitrogen electronic temperature using the present rate coefficients with those using Drawin's rate coefficients. The figures show that the $N$ atoms are almost completely ionized when the gases reach equilibrium at $30,000 \mathrm{~K}$, and using the new quantal rate coefficients results in a longer time to reach equilibrium than using Drawin's formula. Drawin's formula is an empirical formula assuming the excitation cross section has approximately the same energy dependence as the ionization cross section. This assumption completely fails for exchange collisions. (See Eq. (21)). It is therefore not surprising that the result of the mole fraction calculations using quantal rate coefficients and Drawin rate coefficients differ significantly. We also observe that $T^{e l}$ exhibits a steady state for a very short period of time before reaching equilibrium with the translational temperature. 


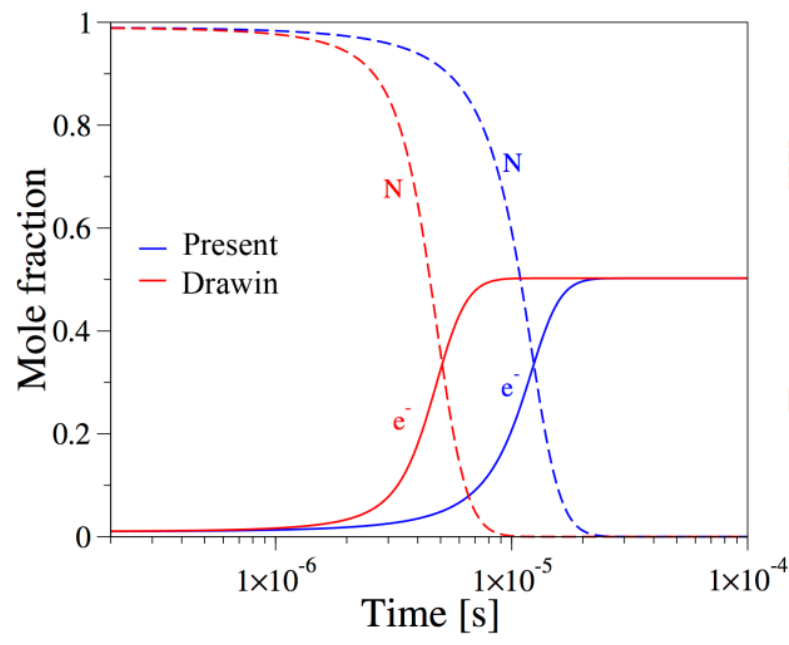

(a)

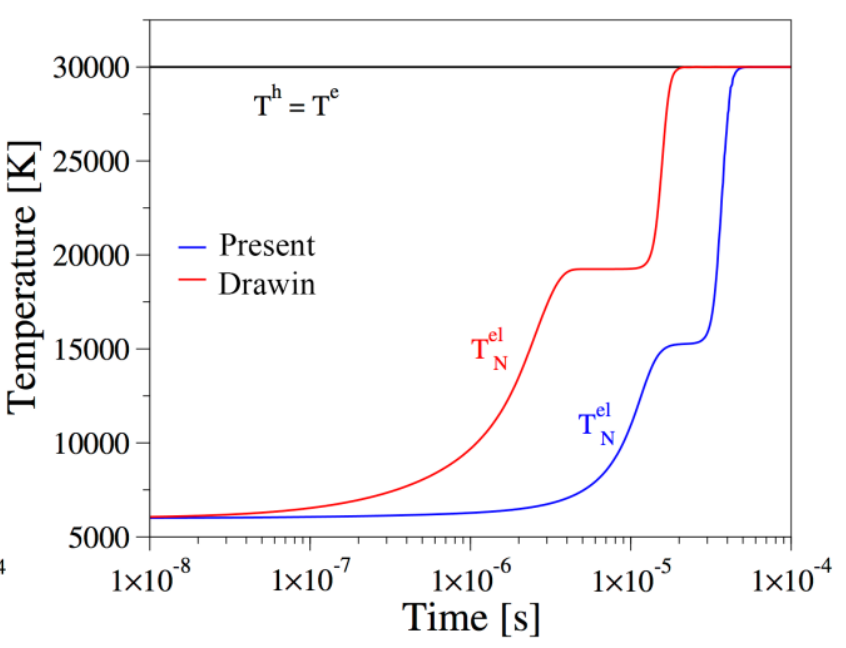

(b)

Figure 6. Comparison of time evolution of (a) mole fractions and (b) electronic temperature using the present and Drawin's rate coefficients for nitrogen atoms undergoing internal excitation and ionization in an isothermal and isochoric heat bath.

\section{B. Thermal and chemical non-equilibrium processes in a nitrogen gas mixture passing through a normal} shock

In the second test case, we consider a more complicated gas mixture, consisting of nitrogen molecules, atoms, ions, and electrons. Behind a strong shock, the gas may encounter the following collisional processes involving heavy particle- and electron-impact excitation, dissociation, and ionization:

- $\quad$ heavy particle-impact excitation

$$
\begin{aligned}
N_{2}^{i}+N_{2}^{j} & \Leftrightarrow N_{2}^{k}+N_{2}^{l} \\
N_{2}^{i}+N^{p} & \Leftrightarrow N_{2}^{k}+N^{r} \\
N^{p}+N^{q} & \Leftrightarrow N^{r}+N^{s}
\end{aligned}
$$

- heavy particle-impact dissociation

$$
\begin{aligned}
N_{2}^{i}+N_{2}^{j} & \Leftrightarrow N_{2}^{k}+N^{r}+N^{s} \\
N_{2}^{i}+N^{p} & \Leftrightarrow N^{r}+N^{s}+N^{t}
\end{aligned}
$$

- heavy particle-impact ionization

$$
N^{p}+N^{q} \Leftrightarrow N_{2}^{+r}+e
$$

- electron-impact excitation

$$
\begin{aligned}
& N_{2}^{i}+e \Leftrightarrow N_{2}^{k}+e \\
& N^{p}+e \Leftrightarrow N^{r}+e
\end{aligned}
$$

- electron-impact dissociation 


$$
N_{2}^{i}+e \Leftrightarrow N^{r}+N^{s}+e
$$

- electron-impact ionization

$$
N^{p}+e \Leftrightarrow N^{+r}+e+e
$$

Here the superscripts $i, j, k, l, p, q, r, s$, and $t$ denote generic indices for internal states of a species. Since not all the microscopic state-to-state rate coefficients are currently available, we simplify the collisional processes in the present study. For molecules, we include only the vibrational states associated with the ground electronic states $N_{2}\left(X^{1} \begin{array}{l}+ \\ \hline\end{array}\right)$ in processes (25), (26), (28), (29), (31), and (33). We also include the vibrational states associated with the first excited electronic state $N_{2}\left(A^{3}{ }_{u}^{+}\right)$in processes (25) and (26). For atoms, we include the ground state $N\left({ }^{4} S^{o}\right)$ in process (26), (27) (28), (29), (30), and (33), and two metastable states $N\left({ }^{2} D^{o}\right)$ and $N\left({ }^{2} P^{o}\right)$ in processes (27) and (30). In processes (32) and (34), we include all electronic states of $N$. For ions, we include only the ground state $N_{2}^{+}\left(X_{g}^{2}{ }_{g}^{+}, v=0\right)$ in process (30) and $N^{+}\left({ }^{3} P\right)$ in process (34).

In the second test case, we employ the newly calculated rates for processes (32) and (34) in a one-dimensional flow code ${ }^{45}$ to study the evolution of thermal and chemical non-equilibrium processes behind a normal shock for the nitrogen gas mixture described above. The rate coefficients for other processes used in the flow code are obtained from references 45-50. The Euler equations, including the continuity equations for internal energy states coupled with the conservation equations for total momentum, total energy, and electron energy, are solved in space. The gas mixture is assumed to be in chemical and thermal equilibrium upstream of the shock, with pressure equal to $2 \mathrm{~Pa}$, temperature $300 \mathrm{~K}$, and flow speed $10 \mathrm{~km} / \mathrm{s}$. Figure 7 (a) shows the mole fractions of the gas mixture species as a function of distance behind the shock. It can be seen that electrons are first produced by heavy particle associative ionization process (Eq. (30)) and then by electron-impact ionization process (Eq. (34)). Figure 7(b) depicts various temperatures behind the shock. The heavy particle temperature $T^{h}$ and the electron temperature $T^{e}$ are computed directly from simulated energy variables, while the fictitious internal electron and vibrational temperatures $T_{N}^{e l}$ and $T_{N_{2}}^{v}$, for $N$ and $N_{2}$ respectively, are post-processed quantities defined by Eq. (24). From these figures, it is apparent that the cooling of the heavy-particle translational temperature is caused by the excitation of internal modes and by the onset of chemical reactions. The distance required to reach complete equilibrium slightly exceeds $0.1 \mathrm{~m}$ in this case. 


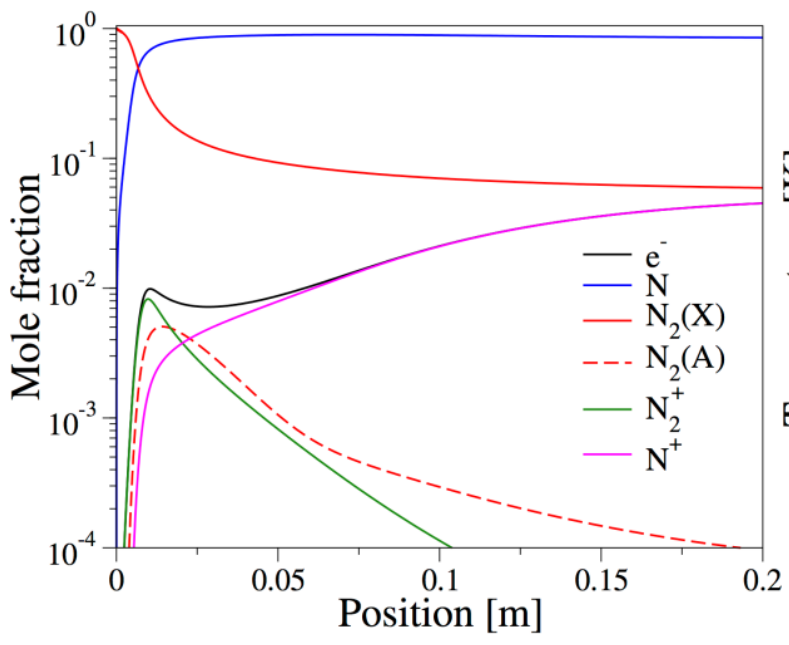

(a)

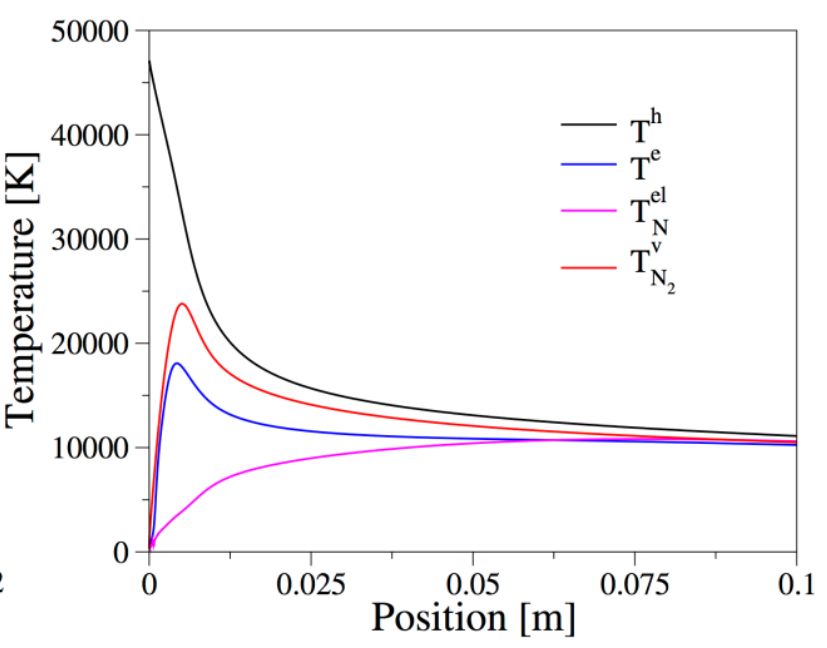

(b)

Figure 7. (a) Mole fraction and (b) temperature distributions of a nitrogen gas mixture passing through a normal shock undergoing heavy particle- and electron-impact excitation, dissociation, and ionization

Acknowledgement

This work was partially funded by NASA ESM program.

\section{$\underline{\text { References }}$}

${ }^{1}$ Panesi, M., Huo, W.M., "Non-equilibrium ionization phenomena behind shock waves", AIAA Paper 2011-3629.

${ }^{2}$ Huo, W.M., Panesi, M., Magin, T.E., "Ionization phenomena behind shock waves" in "Physical Phenomena in

Shock Waves”, Brun, R., ed. Springer-Verlag, Berlin (2012), 149-192.

${ }^{3}$ Park, C., "Review of Chemical-Kinetics Problems of Future NASA Missions, I. Earth Entries", Journal of Thermophysics and Heat Transfer, 7, 385 (1993).

${ }^{4}$ Park, C., Jaffe, R.L., Partridge, H., "Chemical Kinetics Parameters of Hyperbolic Earth Entry”, Journal of Thermophysics and Heat Transfer, 15, 76 (2001).

5 Losev, S.A.; Makarov, V.N.; Pogosbekyan, M.J.; Shatalov, O.P.; SNikol'sky, V., "Thermochemical Nonequilibrium Kinetic Models in Strong Shock Waves on Air" AIAA Paper 94-1900 (1994).

${ }^{6}$ Bird, G.A., Molecular Gas Dynamics and the Direct Simulations of Gas Flows, Clarendon, Oxford (1994).

${ }^{7}$ Bird, G.A., Rarefied Gas Dynamics, Vol. 74, S. Fisher, Editor, New York (1981).

${ }^{8}$ Whiting, E.E., Park, C., Liu, Y., Arnold, J.O., Patterson, J.A.,"NEQAIR96, Nonequilibrium and Equilibrium Radiative Transport and Spectra Program: User's Manual", NASA Reference Publication.

9 Gryzinski, M., "Two-Particle Collisions. II. Coulomb Collisions in the Laboratory Systems of Coordinates", Phys. Rev. A, 138, 322 (1995).

${ }^{10}$ Bultel, A., Chéron, B.G., Bourdon, A., Motapon, O., Schneider, I.F., Phys. Plasmas, 13, 043502 (2006).

${ }^{11}$ H. Drawin, Atomic Cross Sections for Inelastic Electronic Collisions, Association Euratom-CEA. Rept.EURCEA-FC 236, Cadarache, France (1963).

${ }^{12}$ H. Drawin, Zeitschrift fur Physik D: Atoms, Molecules and Clusters 211, 3, 404 (1968).

${ }^{13}$ Hyun, S.Y., Park, C., Chang, K.S., "Rate Parameters for Electronic Excitation of Diatomic Molecules, III. CN Radiation behind a Shock Wave", AIAA Paper 2008-1276 (2008).

${ }^{14}$ Wray, A., "HyperRad: A Code and Database Framework for Hypersonic Entry Radiation", Progress Report presented at the NASA Fundamental Aeronautics annual meeting, Cleveland, March 2011.

${ }^{15}$ Joachain, C.J., Quantum Collision Theory, North-Holland, Amsterdam (1975).

16 http://www.nist.gov/pml/data/asd.cfm

${ }_{17}$ http://nlte.nist.gov/MCHF/view.html

18 http://cdsweb.u-strasbg.fr/topbase/topbase.html

${ }_{19}$ Carbon, D.F., and Huo, W.M., private communication. 
${ }^{20}$ Tayal, S.S., and Zatsarinny, O., "B-spline R-Matrix with Pseudostates Approach for Electron Impact Excitation of Atomic Nitrogen" Jurnal of Physics B 38, 3631-3645 (2005).

${ }^{21}$ Zatsarinny, O., and Tayal, S.S., "Low-Energy Electron Collisions with Atomic Oxygen: R-Matrix Calculation with Non-Orthogaonl Oritals", Journal of Physics B 34 1299-1319 (2001).

${ }^{22}$ Zatsarinny, O., and Tayal, S.S., "R-Matrix Calculation with Non-Orthogaonl Oritals for Electron Impact Excitation of Atomic Oxygen", Journal of Physics B 35 241-253 (2002).

${ }^{23}$ Zatsarinny, O., Bartschat, K., Bandurina, L., and Gedeon, V., "Electron-Impact Excitation of Carbon”, Physical Review A 71, 042702-1 - 042701-9 (2005).

${ }^{24}$ Zatsarinny, O., and Tayal, S.S., "Electron Collisional Excitation Rates for O I Using the B-Spline R-Matrix Approach", The Astrophysical Journal Supplement Series 148, 575-582 (2003).

${ }^{25}$ Frost, R.M., Awakowicz, P., Summers, H.P., and Badnell, N.R. "Calculated Cross Sections and Measured Rate Coefficients for Electron-Impact Excitation of Neutral and Singly Ionized Nitrogen", Journal of Applied Physics 84, 2989-3003 (1998).

${ }^{26}$ Dillon, M.A., and E.N. Lassettre, E.N., "A Collision Cross Section Study of the $1^{1} S \rightarrow 2^{1} P$ and $1{ }^{1} S \rightarrow 2^{1} S$ Transitions in helium at kinetic energies from $200-700 \mathrm{eV}$. Failure of the Born Approximation at Large Mometum Changes", Journal of Chemical Physics 62, 2373 - 2390 (1975).

${ }^{27}$ Lassettre, E.N., Skerbelle, A., and Dillon, M.A., "Generalized Oscillator Strength for $1^{1} S \rightarrow 2^{1} P$ Transition in Helium. Theory of Limiting Oscillator Strengths”, Journal of Chemical Physics 50, 1829 - 1839 (1969).

${ }^{28}$ Rau, A.P., and Fano, U., Physical Review 162, 68 (1967).

${ }^{29}$ Kim, Y.-K., "Scaling of Plane-Wave Born Cross Sections for Electron-Impact Excitation of Neutral Atoms", Physical Review A, 64, 032713-1 - 032713-10 (2001).

30 Doering, J.P., and Goembel, L., "Absolute Differential and Integral electron Excitation Cross Sections for Atomic Nitrogen: 2. The ${ }^{4} S_{0} \rightarrow 2 p^{4}{ }^{4}$ P Transition from 30 to $100 \mathrm{eV}$ ' Journal of Geophysical Research 97, 4295 4298 (1992).

${ }^{31}$ Vaughan, S.O., and Doering, J.P., "Absolute Experimental Differential and Integral Electron Excitation Cross Sections for Atomic Oxygen 4. The $\left(3 p \rightarrow 3 s^{\prime \prime}{ }^{3} P^{o}\right),\left(3 p \rightarrow 2 s 2 p^{5}{ }^{3} P^{o}\right),\left(3 p \rightarrow 4 d^{\prime}{ }^{3} P^{o}\right)$ Autoionizing Transitions $(878,792$, and $770 \AA)$ and Five Members of the $\left(3 p \rightarrow n d^{3} D^{o}\right)$ Rydberg Series (1027 $)$ )", Journal of Geophysical Research, 93, 289-293 (1988).

${ }^{32}$ Kanik, I., Johnson, P.V., Das, M.B., Khakoo, M.A., and Tayal, S.S., "Electron-Impact Studies of Atomic Oxygen: I. Differential and Integral Cross Sections; Experimental and Theory”, Journal of Physics B 34, 2647-2665 (2001).

${ }^{33}$ Vaughan, S.O., and Doering, J.P., "Absolute Experimental Differential and Integral Electron Excitation Cross Sections for Atomic Oxygen 3. The $\left({ }^{3} \mathrm{P} \rightarrow{ }^{3} D^{o}\right)$ Transition (989 A) from 20 to $200 \mathrm{eV}$ with Improved Values for the $\left({ }^{3} P \rightarrow{ }^{3} S^{o}\right)$ Transition (1304 ̊)", Journal of Geophysical Research, 92, 7749-7752 (1987).

${ }^{34}$ Gulcicek, E.E., and Doering, J.P., "Absolute Differential and Integral Electron Excitation Cross Sections for Atomic Oxygen 5. Revised Values for the ${ }^{3} P \rightarrow{ }^{3} S^{o}(1304 \AA)$ and the ${ }^{3} P \rightarrow{ }^{3} D^{o}(989 \AA)$ Transitions below $30 \mathrm{eV}$ ", Journal of Geophysical Research, 93, 5879-5884 (1988).

${ }^{35}$ Zatsarinny, O., Bartschat, K., Bandurina, L., and Gedeon, V., "Electron-Impact Excitation of Carbon”, Physical Review A 71, 042702-1 - 042702-9 (2005).

${ }^{36}$ Ochkur, V. I., "The Born-Oppenheimer Method in the Theory of Atomic Collisions", Soviet Physics JETP 18, 503-508 (1964).

${ }^{37}$ Rudge, M.R. H., "The Calculation of Exchange Scattering Amplitudes", Proceeding of the Physical Society, 85, 607-608 (1965).

${ }^{38}$ Kim, Y.K., "Scaling of Plane-Wave Born Cross Sections for Electron-Impact Excitation of Neutral Atoms", Physical Review A 64, 032713-1 - 032713-10 (2001).

39 Gallagher, T.F., "Rydberg Atoms" Chapter 14 in Springer Handbooks of Atomic, Molecular, and Optical Physics, Drake, G.W.F., Editor, p.235-245 (2005)

${ }^{40}$ Frost, R.M., Awakowicz, P., Summers, H.P., and Badnell, N.R., "Calculated Cross Sections and Measured Rate Coefficients for Electron-Impact Excitation of Neutral and Singly Ionized Nitrogen", Journal of Applied Physics 84, 2989-3003 (1998).

${ }^{41}$ Liu, Y., Huo, W.M., Wray, A., and Carbon, D.F., "Electron Stark Broadening Database for Atomic N, $O$, and C Lines", AIAA paper 2012-2739, 2012.

${ }^{42}$ Huo, W.M., "Electron-impact Excitation and Ionization in Air", VKI Special Course on Non-Equilibrium Gas Dynamics, from Physical Models to Hypersonic Flights. von Karman Inst. For Fluid Dynamics, Rhode-SaintGenèse, Belgium, September (2008). 
${ }^{43}$ Radhakrishnan, K., and Hindmarsh, A.C., "Description and Use of LSODE, the Livermore Solver for Ordinary Differential Equations," NASA Reference Publication 1327, 1993. Also available online: https://computation.llnl.gov/casc/odepack/download/lsode_agree.html.

${ }^{44}$ Liu, Y., Panesi, M., Sahai, A., and Vinokur, M., "General Multi-Group Macroscopic Modeling for ThermoChemical Non-Equilibrium Gas Mixtures," AIAA paper 2014-3205, 2014.

${ }^{45}$ Heritier, K.L., Jaffe, R.L., Laporta, V., and Panesi, M., "Energy Transfer Models in Nitrogen Plasmas: Analysis of $N_{2}\left(X^{1}{ }_{g}^{+}\right)-N\left({ }^{4} S_{u}\right)-e$ Interaction," Journal of Chemical Physics 141, 184302 (2014).

${ }^{46}$ Adamovich, I. V., Macheret, S.O., Treanor, C.E., "Vibrational Energy Transfer Rates Using a Forced Harmonic Oscillator Model," Joutnal of Thermophysics and Heat Transfer 12 (1992).

${ }^{47}$ Jaffe, R., Schwenke, D.W., Chaban, G., and Huo, W.M., "Vibrational and Rotational Excitation and Relaxation of Nitrogen from Accurate Theoretical Calculations," AIAA paper 2008-1208, 2008.

${ }^{48}$ Chaban, G., Jaffe, R., Schwenke, D.W., and Huo, W.M., "Dissociation Cross Sections and Rate Coefficients for Nitrogen From Accurate Theoretical Calculations," AIAA paper 2008-1209, 2008.

${ }^{49}$ Bacri, J., and Medani, A., "Electron Diatomic Molecule Weighted Total Cross Section Calculation, I. Principles for Calculation," Physica 101C, 399-409 (1980).

${ }^{50}$ Bacri, J., and Medani, A., "Electron Diatomic Molecule Weighted Total Cross Section Calculation, II. Application to the Nitrogen Molecule," Physica 101C, 410-419 (1980). 